\title{
Renormalization-group analysis for low-energy scattering of charged particles
}

\author{
Shung-ichi Andd* and Michael C. Birseむt \\ Theoretical Physics Group, School of Physics and Astronomy, \\ The University of Manchester, Manchester, M13 9PL, UK
}

\begin{abstract}
The low-energy scattering of two charged particles is analyzed using a renormalization group approach based on dimensional regularization with power-divergence subtraction. A nontrivial solution with a marginally unstable direction is found, corresponding to a system with a bound state at zero energy. For purely energy-dependent perturbations around this solution, the power counting agrees with that from Wilsonian methods. These terms in the effective potential are in direct correspondence with the the terms in the Coulomb-distorted effective-range expansion. We also study perturbations that depend on off-shell momenta as well as energy, and we show that these affect only the off-shell form of the scattering matrix. These terms are of higher order that the corresponding energy-dependent ones and so terms in the potential that depend only on the off-shell momenta do not have definite orders in power counting.
\end{abstract}

*Electronic address: shung-ichi.ando@manchester.ac.uk

${ }^{\dagger}$ Electronic address: mike.birse@manchester.ac.uk 


\section{INTRODUCTION}

The effective field theories (EFTs) that have been developed in recent years are now important tools for analyzing the scattering of low-energy particles, particularly in the context of nuclear forces. (For reviews, see, e.g., Refs. [1, 2, 3].) They can provide systematic expansions of interactions in powers of low-energy scales. In addition, they form a fieldtheoretic framework for extending the older effective-range expansions developed by Bethe and others [4]. In the context of charged particle scattering, Kong and Ravndal [5, 6] showed how Bethe's Coulomb-modified effective-range expansion could be represented as a pionless EFT.

The results of Kong and Ravndal [5, 6] at NLO showed unexpectedly strong dependence of the scattering length on the subtraction scale $\mu$. More recently, this approach was extended to NNLO [7] and similar dependence of other coefficients on $\mu$ were observed, along with dependencies on the renormalization scheme and the choice of momentum structures in the NNLO terms. These results contrast with ones obtained from an analysis using a Wilsonian renormalization group [8], which showed a one-to-one correspondence between terms in the effective potential and the effective-range expansion.

The main difference between the effective short-range potentials used in those two approaches is that those in Refs. [5, 6, 7] depend on the off-shell relative momenta of the two particles whereas those in Ref. [8] depend on energy. Applications of the renormalization group (RG) [9] to pure short-range interactions [10] have shown that, in systems with bound or virtual states close to threshold, momentum- and energy-dependent terms have different scale dependence and hence appear at different orders in the power counting.

To examine whether this differences between these short-range potentials is responsible, we have made an RG analysis of their scaling properties. To facilitate the comparison with Refs. [5, 6, 7] we have used dimensional regularization (DR) with the power divergence subtraction (PDS) [11, 12] and modified minimal subtraction $(\overline{\mathrm{MS}})$ schemes. We consider potentials that can depend on energy to all orders but, for simplicity, we restrict our analysis to the simplest momentum-dependent term, which is of second order in the off-shell momenta.

The paper is organized as follows. In Sec. II, we apply the RG to an energy-dependent short-range interaction in the presence of the Coulomb potential. We concentrate on the 
expansion around a marginally unstable nontrivial solution that is the analogue of the fixed point discussed in Ref. [10]. This allows us to confirm that DR leads to the same RG flow as found in Ref. [8] using a sharp cut-off on the distorted waves. Then, in Sec. III, we find the leading momentum-dependent perturbation around our nontrivial solution. This has a different RG eigenvalue from the corresponding energy-dependent term. Its form suggests that it contributes only to the off-shell dependence of the scattering matrix, and we conform this by calculating the on-shell scattering amplitude in Sec. IV. Finally, we discuss the implications of this and other features of our results in Sec. V.

\section{SCATTERING BY LONG- AND SHORT-RANGE FORCES}

The two-body potential $V$ between two charged particles can be separated into two pieces:

$$
V=V_{C}+V_{S}
$$

where $V_{C}$ is the long-range Coulomb potential and $V_{S}$ is the short-range strong interaction. For momenta that are too low to resolve the range of the short-range interaction, we can represent this part of the potential by delta functions and their derivatives. The RG will then lead us to a systematic power counting for these terms.

For the sum of the two potentials above, the scattering matrix $T$ can be split into $T_{C}$, the pure Coulomb $T$ matrix, and $T_{S C}$ which describes scattering between distorted waves of the Coulomb potential. If we restrict our attention to $S$-waves, the on-shell matrix element of $T_{S C}$ can be related to the strong-interaction phase shift, $\delta_{S C}(p)$, by

$$
\left\langle\psi_{C}^{-}(p)\left|T_{S C}\right| \psi_{C}^{+}(p)\right\rangle=-\frac{4 \pi}{M p} \frac{e^{2 i \sigma_{0}(p)}}{\cot \delta_{S C}(p)-i},
$$

where $\sigma_{0}$ is the Coulomb phase shift, $\left|\psi_{C}^{ \pm}(p)\right\rangle$ are the in- and out-going Coulomb wave functions, and $p=\sqrt{M E}$ is the on-shell momentum. ${ }^{1}$

At low energies, the $S$-wave phase shift $\delta_{S C}(p)$ can be written in the form of an effectiverange expansion as

$$
\mathcal{C}(\kappa / p) p \cot \delta_{S C}(p)+2 \kappa \operatorname{Re}\{H(\kappa / p)\}=-\frac{1}{a_{C}}+\frac{1}{2} r_{0} p^{2}+\cdots
$$

\footnotetext{
${ }^{1}$ Here we are considering scattering of two identical particles of mass $M$. More generally $M$ should be replaced by twice the reduced mass.
} 
where

$$
\mathcal{C}(\eta)=\frac{2 \pi \eta}{e^{2 \pi \eta}-1}, \quad H(\eta)=\psi(i \eta)+\frac{1}{2 i \eta}-\ln (i \eta),
$$

$\kappa$ is the inverse of the Bohr radius (we will give its definition below), and $\psi$ is the logarithmic derivative of the $\Gamma$ function. In this expansion, $a_{C}$ is the scattering length and $r_{0}$ is the effective range.

This $T$ matrix satisfies the Lippmann-Schwinger (LS) equation

$$
T_{S C}(E)=V_{S}+V_{S} G_{C}(E) T_{S C}(E) .
$$

Here $G_{C}=1 /\left(E-H_{0}-V_{C}+i \epsilon\right)$ is the Coulomb Green's function. In terms of the Coulomb wave functions, this has the form

$$
G_{C}(E)=M \int \frac{d^{3} \mathbf{q}}{(2 \pi)^{3}} \frac{\left|\psi_{C}^{+}(q)\right\rangle\left\langle\psi_{C}^{+}(q)\right|}{p^{2}-q^{2}+i \epsilon} .
$$

\section{RG ANALYSIS FOR ENERGY-DEPENDENT POTENTIALS}

Before examining momentum-dependent short-range potentials, we first consider purely energy-dependent ones. This analysis reproduces the results of Ref. [8], but within the framework of DR and the PDS scheme. This establishes the notation we shall use for more general potentials and it allows us to make direct contact with the approach of Kong and Ravndal [5, 6], which was also used in Ref. [7].

In DR, we replace the 3+1-dimensional Coulomb Green's function by

$$
G_{C}(E, \mu)=M\left(\frac{\mu}{2}\right)^{4-d} \int \frac{d^{d-1} \mathbf{q}}{(2 \pi)^{d-1}} \frac{\left|\psi_{C}^{+}(q)\right\rangle\left\langle\psi_{C}^{+}(q)\right|}{p^{2}-q^{2}+i \epsilon} .
$$

The resulting integrals have poles at $d=3$ and 4 , corresponding to linear and logarithmic divergences respectively. We subtract all of these, using the $\overline{\mathrm{MS}}$ scheme for the poles at $d=4$.

For a simple $\delta$-function interaction, its strength $V_{S}(p, \kappa, \mu)$ can depend on three scales: the on-shell momentum $p$, the inverse of the Bohr radius,

$$
\kappa=\frac{\alpha M}{2},
$$

and the subtraction scale $\mu$. The resulting regularized LS equation for $T_{S C}$ takes the form

$$
T_{S C}(p, \kappa)=V_{S}(p, \kappa, \mu)+V_{S}(p, \kappa, \mu) J_{0}^{\overline{\mathrm{MS}}}(p, \kappa, \mu) T_{S C}(p, \kappa),
$$


where the bubble integral is defined by

$$
J_{0}=\int \frac{d^{3} \mathbf{k}}{(2 \pi)^{3}} \frac{d^{3} \mathbf{k}^{\prime}}{(2 \pi)^{3}}\left\langle\mathbf{k}\left|G_{C}\right| \mathbf{k}^{\prime}\right\rangle .
$$

In the dimensional regularization scheme used here, this becomes [6, 7]

$$
J_{0}^{\overline{\mathrm{MS}}}(p, \kappa, \mu)=-\frac{M}{4 \pi} \mu+\frac{\kappa M}{2 \pi}\left[1-\gamma+\ln \left(\frac{\mu}{4 \kappa}\right)\right]-\frac{\kappa M}{2 \pi} H(\eta),
$$

where $\gamma$ is the Euler-Mascheroni constant. Note that the Coulomb wave functions at the origin, $\int d^{3} \mathbf{k} /(2 \pi)^{3}\left\langle\mathbf{k} \mid \psi_{C}(q)\right\rangle$, are finite and so the only divergences here arise from the integral over q. With momentum-dependent interactions, we encounter the derivatives of these functions. These diverge at the origin and so require additional regularization, as discussed in the next section.

We now demand that the $T$ matrix be independent of the arbitrary scale $\mu$. This condition ensures that physical scattering observables will not depend on $\mu$. It leads to a differential equation for the effective potential, which is similar to that controlling the cut-off dependence of the potential in Wilsonian approaches [8, 10]. This equation has the form

$$
\frac{\partial}{\partial \mu} V_{S}(p, \kappa, \mu)=-V_{S}(p, \kappa, \mu)^{2} \frac{\partial}{\partial \mu} J_{0}^{\overline{\mathrm{MS}}}(p, \kappa, \mu)=-V_{S}(p, \kappa, \mu)^{2}\left(-\frac{M}{4 \pi}+\frac{M}{2 \pi} \frac{\kappa}{\mu}\right) .
$$

This equation can be converted into an RG equation by expressing all dimensioned quantities in units of $\mu$. We define $\hat{p}=p / \mu$ and $\hat{\kappa}=\kappa / \mu$ and introduce the rescaled potential

$$
\hat{V}_{S}(\hat{p}, \hat{\kappa}, \mu)=\frac{M \mu}{4 \pi} V_{S}(\mu \hat{p}, \mu \hat{\kappa}, \mu) .
$$

In terms of these, we can rewrite Eq. (12) as

$$
\mu \frac{\partial}{\partial \mu}\left(\frac{1}{\hat{V}_{S}}\right)=\hat{p} \frac{\partial}{\partial \hat{p}}\left(\frac{1}{\hat{V}_{S}}\right)+\hat{\kappa} \frac{\partial}{\partial \hat{\kappa}}\left(\frac{1}{\hat{V}_{S}}\right)-\frac{1}{\hat{V}_{S}}-(1-2 \hat{\kappa}) .
$$

Below all production thresholds, the effective potential should be an analytic function of the energy and all other low-energy scales. We thus require solutions to this equation to be analytic in $\hat{p}^{2}$ and $\hat{\kappa}$.

Fixed points of the RG equation play a crucial role, as discussed in detail in Ref. [10]. In the limit $\mu \rightarrow 0$, the rescaled potential, being dimensionless, is expected to become independent of $\mu$. In other words, $\hat{V}_{S}$ should flow towards an infrared fixed point as $\mu \rightarrow 0$.

The only true fixed-point solution to Eq. (14) is the trivial one, $\hat{V}_{0}=0$. Although there is no other solution that is independent of $\mu$ and analytic in $\hat{\kappa}$, there is a marginally unstable 
solution that flows logarithmically towards the trivial point. This behavior is the same as was found in [8] using a sharp cut-off on the basis of distorted waves. In the current scheme, it has the form

$$
\frac{1}{\hat{V}_{0}(\hat{\kappa}, \mu)}=-1+2 \hat{\kappa} \ln \left(\frac{\mu}{\Lambda}\right)
$$

where $\Lambda$ is another arbitrary scale.

We now consider perturbations around this potential that scale with definite powers of $\mu$. Taking a single term of this form,

$$
\frac{1}{\hat{V}_{S}(\hat{p}, \hat{\kappa}, \mu)}=\frac{1}{\hat{V}_{0}(\hat{\kappa}, \mu)}-C \mu^{\nu} f(\hat{p}, \hat{\kappa}),
$$

and inserting it into the RG equation (14), we find that $f(\hat{p}, \hat{\kappa})$ satisfies the eigenvalue equation

$$
\nu f=\hat{p} \frac{\partial f}{\partial \hat{p}}+\hat{\kappa} \frac{\partial f}{\partial \hat{\kappa}}-f .
$$

Solutions to this are just products of powers of the low-energy scales,

$$
f(\hat{p}, \hat{\kappa})=\hat{p}^{2 m} \hat{\kappa}^{n}
$$

and their eigenvalues are

$$
\nu=2 m+n-1
$$

These include one unstable perturbation with a negative eigenvalue $(m=n=0)$, just as in the expansion around the nontrivial fixed point of the pure short-range potential [10]. There is also a marginal perturbation with $\nu=0(m=0, n=1)$ as expected from the logarithmic dependence on $\mu$ noted above. In the corresponding power counting, these terms can be assigned orders $d=\nu-1=2 m+n-2$.

The full short-range potential, expanded around the nontrivial solution $\hat{V}_{0}$, is given by

$$
\frac{1}{\hat{V}_{S}(\hat{p}, \hat{\kappa}, \mu)}=\frac{1}{\hat{V}_{0}(\hat{\kappa}, \mu)}-\sum_{m, n \geq 0} C_{m n 0} \mu^{2 m+n-1} \hat{p}^{2 m} \hat{\kappa}^{n} .
$$

The coefficient of the marginal perturbation, $C_{010}(\Lambda)$ depends logarithmically on $\Lambda$ so that the full potential is independent of that arbitrary scale.

To interpret the terms in this potential, we solve the LS equation with it and calculate the on-shell $T$ matrix. Returning to physical units, the potential becomes

$$
\frac{1}{V_{S}(p, \kappa, \mu)}=\frac{1}{V_{0}(\kappa, \mu)}-\frac{M}{4 \pi} \sum_{m, n \geq 0} C_{m n 0} p^{2 m} \kappa^{n},
$$


where

$$
\frac{1}{V_{0}(\kappa, \mu)}=\frac{M}{4 \pi}\left[-\mu+2 \kappa \ln \left(\frac{\mu}{\Lambda}\right)\right]
$$

The corresponding scattering amplitude is

$$
\left\langle\psi_{C}^{-}(p)\left|T_{S C}\right| \psi_{C}^{+}(p)\right\rangle=\frac{e^{2 i \sigma_{0}} \mathcal{C}(\kappa / p)}{\frac{1}{V_{S}(p, \kappa, \mu)}-J_{0}^{\overline{\mathrm{MS}}}(p, \kappa, \mu)} .
$$

This leads to

$$
\mathcal{C}(\kappa / p) p \cot \delta_{S C}+2 \kappa \operatorname{Re}\{H(\kappa / p)\}=\sum_{m, n \geq 0} C_{m n 0} p^{2 m} \kappa^{n}+2 \kappa\left[1-\gamma+\ln \left(\frac{\Lambda}{4 \kappa}\right)\right]
$$

since the imaginary part of $H$ is

$$
\operatorname{Im}\{H(\eta)\}=\frac{\mathcal{C}(\eta)}{2 \eta}
$$

Comparing this with the Coulomb effective-range expansion, Eq. (3), we see that the coefficients of the energy-dependent perturbations are directly related the terms in that expansion by

$$
\begin{aligned}
-\frac{1}{a_{C}} & =C_{000}+C_{010}(\Lambda) \kappa+2 \kappa\left[1-\gamma+\ln \left(\frac{\Lambda}{4 \kappa}\right)\right]+\mathcal{O}\left(\kappa^{2}\right), \\
\frac{1}{2} r_{0} & =C_{100}+C_{110} \kappa+\mathcal{O}\left(\kappa^{2}\right) .
\end{aligned}
$$

If the coefficients in the potential are fine-tuned such that $1 / V_{0}$ exactly cancels the real part of $J_{0}^{\overline{\mathrm{MS}}}$ then the $T$ matrix develops a pole at $p=0$, corresponding to a zero-energy bound state. This is the scale-free system, analogous to the one corresponding to the fixed point in Ref. [10]. In the present case it is unstable against introducing a nonzero value for $C_{000}$ or changing $C_{010}$ from the value that gives a vanishing coefficient for $\kappa$.

The full expansion around this point is a double one in powers of the energy (or $p^{2}$ ) and $\alpha$ (or $\kappa)$. For two protons $\kappa$ is approximately $3 \mathrm{MeV}$. This is small compared to $m_{\pi}$ and so this power counting can be used in the context of a pionless effective theory. More generally this scale is given by $\kappa=Z_{1} Z_{2} \alpha M_{r}$, where $M_{r}$ is the reduced mass and the expansion in powers of $\kappa$ can break down if it is comparable to the scales of the underlying physics. For example, $\kappa$ for the system of two $\alpha$ particles is about $60 \mathrm{MeV}$, and so is of the order of $m_{\pi}$. In such a case, $\kappa$ can be treated as a high-energy scale and the resulting pionless effective theory can be expanded in powers of energy only, as pointed out by Higa et al. [13]. 


\section{RG ANALYSIS FOR MOMENTUM-DEPENDENT POTENTIAL}

We now turn to more general short-distance potentials that depend on momenta as well as energy. For simplicity, we consider potentials of the form

$$
V_{S}\left(p, \kappa, k^{\prime}, k, \mu\right)=V_{1}(p, \kappa, \mu)+V_{2}(p, \kappa, \mu) k^{2},
$$

where $V_{1}$ and $V_{2}$ are energy-dependent functions and $k$ is the initial relative momentum. Although this expression is not Hermitian, it can easily be made so by adding a matching term with $k \rightarrow k^{\prime}$.

If we write the corresponding (off-shell) $T$ matrix as

$$
T_{S C}=T_{1}+T_{2} k^{2}
$$

the (regularized) LS equation becomes two coupled linear equations

$$
\begin{aligned}
& T_{1}=V_{1}+V_{1} J_{0}^{\overline{\mathrm{MS}}} T_{1}+V_{2} J_{2}^{\overline{\mathrm{MS}}} T_{1}, \\
& T_{2}=V_{2}+V_{1} J_{0}^{\overline{\mathrm{MS}}} T_{2}+V_{2} J_{2}^{\overline{\mathrm{MS}}} T_{2} .
\end{aligned}
$$

Here $J_{0}$ is the bubble integral defined above and

$$
J_{2}=\int \frac{d^{3} \mathbf{k}}{(2 \pi)^{3}} \frac{d^{3} \mathbf{k}^{\prime}}{(2 \pi)^{3}} k^{2}\left\langle\mathbf{k}\left|G_{C}\right| \mathbf{k}^{\prime}\right\rangle .
$$

We apply DR and PDS to the integrals over $\mathbf{q}$ in the same way for both $J_{0}$ and $J_{2}$. However $J_{2}$ contains an additional divergence, in the integral over $\mathbf{k}$. This is because the Coulomb potential leads to wave functions whose derivatives are singular at the origin. We use DR and PDS for this too but with a different scale, $\lambda$. Using the same scale to regulate all the integrals leads to an $\mathrm{RG}$ equation that contains nonanalytic dependence on the low-energy scales and is not suitable for a scaling analysis. The second scale $\lambda$ can be regarded as analogous to the factorization scale used in parton distributions to separate off the nonperturbative regime of QCD. Although the factorization scale can, and often is, taken to be equal to the renormalization scale, this is not a requirement. The Wilsonian approach to scattering in Ref. [8] used a $\delta$-shell form for the short-range potential to avoid the nonperturbative singularities of several long-range potentials. The resulting physical amplitudes did not depend on the radius chosen for this. In the present case, results should be independent of the new scale $\lambda$, and this will provide an important consistency check on our treatment. 
With this regularization, we have

$$
J_{2}^{\overline{\mathrm{MS}}}=\left(p^{2}-2 \kappa^{2}-2 \kappa \lambda\right) J_{0}^{\overline{\mathrm{MS}}}-\Delta J_{2}
$$

where $J_{0}^{\overline{\mathrm{MS}}}$ is given by Eq. (11) and

$$
\Delta J_{2}=\frac{\pi M}{12} \kappa^{2} \mu+2 \pi M \kappa^{3} \zeta^{\prime}(-2),
$$

and the derivative of the Riemann zeta function is $\zeta^{\prime}(-2)=-0.0304 \cdots$.

By taking appropriate linear combinations of the coupled equations for $T_{1,2}$, we can rewrite them as equations that each involve only one piece of the potential, ${ }^{2}$

$$
\begin{aligned}
& T_{1}=V_{1}\left(1+J_{0}^{\overline{\mathrm{MS}}} T_{1}+J_{2}^{\overline{\mathrm{MS}}} T_{2}\right), \\
& T_{2}=V_{2}\left(1+J_{0}^{\overline{\mathrm{MS}}} T_{1}+J_{2}^{\overline{\mathrm{MS}}} T_{2}\right) .
\end{aligned}
$$

Demanding that the $T$ matrix be independent of $\mu, \partial T_{1,2} / \partial \mu=0$, now leads to

$$
\begin{aligned}
& 0=\frac{\partial V_{1}}{\partial \mu}\left(1+J_{0}^{\overline{\mathrm{MS}}} T_{1}+J_{2}^{\overline{\mathrm{MS}}} T_{2}\right)+V_{1} \frac{\partial J_{0}^{\overline{\mathrm{MS}}}}{\partial \mu} T_{1}+V_{1} \frac{\partial J_{2}^{\overline{\mathrm{MS}}}}{\partial \mu} T_{2}, \\
& 0=\frac{\partial V_{2}}{\partial \mu}\left(1+J_{0}^{\overline{\mathrm{MS}}} T_{1}+J_{2}^{\overline{\mathrm{MS}}} T_{2}\right)+V_{2} \frac{\partial J_{0}^{\overline{\mathrm{MS}}}}{\partial \mu} T_{1}+V_{2} \frac{\partial J_{2}^{\overline{\mathrm{MS}}}}{\partial \mu} T_{2} .
\end{aligned}
$$

Multiplying these from the right by the factor $\left(1+J_{0}^{\overline{\mathrm{MS}}} T_{1}+J_{2}^{\overline{\mathrm{MS}}} T_{2}\right)^{-1}$ and using Eq. (33) for $T_{1,2}$, we arrive at two coupled differential equations for $V_{1,2}$ :

$$
\begin{aligned}
& \frac{\partial V_{1}}{\partial \mu}=-V_{1}^{2} \frac{\partial J_{0}^{\overline{\mathrm{MS}}}}{\partial \mu}-V_{1} V_{2} \frac{\partial J_{2}^{\overline{\mathrm{MS}}}}{\partial \mu} \\
& \frac{\partial V_{2}}{\partial \mu}=-V_{1} V_{2} \frac{\partial J_{0}^{\overline{\mathrm{MS}}}}{\partial \mu}-V_{2}^{2} \frac{\partial J_{2}^{\overline{\mathrm{MS}}}}{\partial \mu} .
\end{aligned}
$$

We rescale the potential as before, defining

$$
\hat{V}_{1}(\hat{p}, \hat{\kappa}, \mu)=\frac{M \mu}{4 \pi} V_{1}(\mu \hat{p}, \mu \hat{\kappa}, \mu), \quad \hat{V}_{2}(\hat{p}, \hat{\kappa}, \mu)=\frac{M \mu^{3}}{4 \pi} V_{2}(\mu \hat{p}, \mu \hat{\kappa}, \mu) .
$$

The rescaled functions $\hat{V}_{1,2}$ then satisfy the coupled RG equations

$$
\mu \frac{\partial}{\partial \mu} \hat{V}_{1}=\hat{p} \frac{\partial}{\partial \hat{p}} \hat{V}_{1}+\hat{\kappa} \frac{\partial}{\partial \hat{\kappa}} \hat{V}_{1}+\hat{V}_{1}+(1-2 \hat{\kappa}) \hat{V}_{1}^{2}
$$

\footnotetext{
${ }^{2}$ These are also easily obtained from the LS equation in the form $T_{S C}=V_{S}+T_{S C} G_{C} V_{S}$, instead of Eq. (5).
} 


$$
\begin{aligned}
& +\left[(1-2 \hat{\kappa})\left(\hat{p}^{2}-2 \hat{\kappa}^{2}-2 \hat{\kappa} \frac{\lambda}{\mu}\right)+\frac{\pi^{2}}{3} \hat{\kappa}^{2}\right] \hat{V}_{1} \hat{V}_{2}, \\
\mu \frac{\partial}{\partial \mu} \hat{V}_{2}= & \hat{p} \frac{\partial}{\partial \hat{p}} \hat{V}_{2}+\hat{\kappa} \frac{\partial}{\partial \hat{\kappa}} \hat{V}_{2}+3 \hat{V}_{2}+(1-2 \hat{\kappa}) \hat{V}_{1} \hat{V}_{2} \\
& +\left[(1-2 \hat{\kappa})\left(\hat{p}^{2}-2 \hat{\kappa}^{2}-2 \hat{\kappa} \frac{\lambda}{\mu}\right)+\frac{\pi^{2}}{3} \hat{\kappa}^{2}\right] \hat{V}_{2}^{2} .
\end{aligned}
$$

We are interested in perturbations around the nontrivial solution described in the previous section, so we expand the potential around $\hat{V}_{0}$ as

$$
\hat{V}_{1}=\hat{V}_{0}+\delta \hat{V}_{1}
$$

and keep terms to first order in $\delta \hat{V}_{1}$ and $\hat{V}_{2}$. The resulting linearized RG equations are

$$
\begin{aligned}
\mu \frac{\partial}{\partial \mu} \delta \hat{V}_{1} \simeq & {\left[\hat{p} \frac{\partial}{\partial \hat{p}}+\hat{\kappa} \frac{\partial}{\partial \hat{\kappa}}+1+2(1-2 \hat{\kappa}) \hat{V}_{0}\right] \delta \hat{V}_{1} } \\
& +\left[(1-2 \hat{\kappa})\left(\hat{p}^{2}-2 \hat{\kappa}^{2}-2 \hat{\kappa} \frac{\lambda}{\mu}\right)+\frac{\pi^{2}}{3} \hat{\kappa}^{2}\right] \hat{V}_{0} \hat{V}_{2}, \\
\mu \frac{\partial}{\partial \mu} \hat{V}_{2} \simeq & {\left[\hat{p} \frac{\partial}{\partial \hat{p}}+\hat{\kappa} \frac{\partial}{\partial \hat{\kappa}}+3+(1-2 \hat{\kappa}) \hat{V}_{0}\right] \hat{V}_{2} . }
\end{aligned}
$$

The second of these is a homogeneous equation for $\hat{V}_{2}$ which does not involve $\delta \hat{V}_{1}$. By comparing it with the equation satisfied by $\hat{V}_{0}$, we find that it has a solution

$$
\hat{V}_{2}=C_{001} \mu^{2} \hat{V}_{0}
$$

with RG eigenvalue $\nu=2$. Other solutions with larger eigenvalues can be obtained by multiplying this by powers of $\hat{p}^{2}$ and $\hat{\kappa}$.

Inserting this solution for $\hat{V}_{2}$ into Eq. (40), we get an inhomogeneous equation for $\delta \hat{V}_{1}$. This has the solution

$$
\delta \hat{V}_{1}=-C_{001} \mu^{2}\left[\left(\hat{p}^{2}-2 \hat{\kappa}^{2}-2 \hat{\kappa} \frac{\lambda}{\mu}\right) \hat{V}_{0}-\frac{\pi^{2}}{3} \hat{\kappa}^{2} \hat{V}_{0}^{2}\right]+\mu^{2}\left(A \hat{\kappa}^{3}+B \hat{\kappa} \hat{p}^{2}\right) \hat{V}_{0}^{2} .
$$

The final term here is a solution to the homogeneous part of the equation, with the same RG eigenvalue as $\hat{V}_{2}$. It corresponds to two of the terms in Eq. (20) when that is expanded to first in order in deviations from $\hat{V}_{0}$. The full momentum-dependent perturbation has the form

$$
\delta \hat{V}_{S}=C_{001} \mu^{2}\left[\left(\hat{k}^{2}-\hat{p}^{2}+2 \hat{\kappa}^{2}+2 \hat{\kappa} \frac{\lambda}{\mu}\right) \hat{V}_{0}+\frac{\pi^{2}}{3} \hat{\kappa}^{2} \hat{V}_{0}^{2}\right]+\mu^{2}\left(A \hat{\kappa}^{3}+B \hat{\kappa} \hat{p}^{2}\right) \hat{V}_{0}^{2} .
$$


As already noted a Hermitian potential can be formed by adding the same structure with $k$ replaced by $k^{\prime}$.

It is worth commenting on several aspects of this term in the potential. First, having solved the RG equation for fixed "factorization scale" $\lambda$, we are now free to choose $\lambda=\mu$ so that the whole expression is proportional to $\mu^{2}$. The RG eigenvalue is $\nu=2$ and so the term is of higher order than the corresponding energy-dependent one, $C_{100} \mu \hat{p}^{2}$ (which has $\nu=1$ ). This is the same pattern as was observed in Ref. [10] for perturbations around the nontrivial fixed point of a pure short-range potential. As in that system, the momentum-dependent perturbation contains the structure $k^{2}-p^{2}$ plus terms arising from the potential. This form suggests that it will vanish when acting on on-shell wave functions and hence it will alter only the off-shell behavior of the $T$ matrix but not observables. We shall see below that this is indeed the case.

\section{SCATTERING BY MOMENTUM-DEPENDENT POTENTIAL}

We now take the momentum-dependent perturbation constructed in the previous section and combine it with the energy-dependent potential of Eq. (21), which we now denote by $V_{S E}$. Working in physical units, the potential has the form in Eq. (27), with

$$
V_{1}=V_{S E}+\delta V_{1}, \quad V_{2}=C_{001} V_{0},
$$

where

$$
\delta V_{1}=-C_{001}\left[\left(p^{2}-2 \kappa^{2}-2 \lambda \kappa\right) V_{0}-\frac{\pi^{2}}{3} \mu \kappa^{2} \frac{M}{4 \pi} V_{0}^{2}\right]+\left(A \kappa^{3}+B \kappa p^{2}\right) \frac{M}{4 \pi} V_{0}^{2} .
$$

Using the LS equation in the form of Eq. (29), we can now calculate the $T$ matrix. Expanded to first order in $\delta V_{1}$ and $V_{2}$, this gives

$$
\begin{aligned}
& T_{1} \simeq \frac{1}{1 / V_{S E}-J_{0}^{\overline{\mathrm{MS}}}}+\left(\frac{1}{1 / V_{S E}-J_{0}^{\overline{\mathrm{MS}}}}\right)^{2}\left(\frac{\delta V_{1}}{V_{S E}^{2}}+\frac{V_{2}}{V_{S E}} J_{2}^{\overline{\mathrm{MS}}}\right), \\
& T_{2} \simeq \frac{1}{1 / V_{S E}-J_{0}^{\overline{\mathrm{MS}}}} \frac{V_{2}}{V_{S E}} .
\end{aligned}
$$

The on-shell $T$ matrix can be written in terms of these as

$$
\left\langle\psi_{C}^{-}(p)\left|T_{S C}\right| \psi_{C}^{+}(p)\right\rangle=\psi_{0}^{2} T_{1}+\psi_{0} \psi_{2} T_{2}
$$


where, following Refs. [5, 6, 7], we have introduced

$$
\begin{aligned}
& \psi_{0}=\int \frac{d^{3} \mathbf{k}}{(2 \pi)^{3}}\left\langle\mathbf{k} \mid \psi_{C}^{+}(p)\right\rangle=\sqrt{\mathcal{C}(\kappa / p)} e^{i \sigma_{0}} \\
& \psi_{2}=\int \frac{d^{3} \mathbf{k}}{(2 \pi)^{3}} k^{2}\left\langle\mathbf{k} \mid \psi_{C}^{+}(p)\right\rangle=\left(p^{2}-2 \kappa^{2}-2 \lambda \kappa\right) \psi_{0} .
\end{aligned}
$$

Note that we have used DR to regularise the divergent derivative of the wave function in $\psi_{2}$, with the same scale $\lambda$ as used above for the similar divergence in $J_{2}$. Inserting the expressions for $T_{1,2}$ from our potential gives

$$
\begin{aligned}
& \frac{e^{-2 i \sigma_{0}}}{\mathcal{C}(\kappa / p)}\left\langle\psi_{C}^{-}(p)\left|T_{S C}\right| \psi_{C}^{+}(p)\right\rangle \\
& \quad \simeq \frac{1}{1 / V_{S E}-J_{0}^{\overline{\mathrm{MS}}}}+\left(\frac{1}{1 / V_{S E}-J_{0}^{\overline{\mathrm{MS}}}}\right)^{2}\left(\frac{\delta V_{1}}{V_{S E}^{2}}+\frac{V_{2}}{V_{S E}^{2}} \frac{\psi_{2}}{\psi_{0}}-\frac{V_{2}}{V_{S E}} \Delta J_{2}\right)
\end{aligned}
$$

where we have used the relation $J_{2} \psi_{0}-J_{0} \psi_{2}=-\Delta J_{2} \psi_{0}$.

If we now substitute our explicit expressions for $\delta V_{1}$ and $V_{2}$, we get

$$
\begin{aligned}
& \frac{e^{-2 i \sigma_{0}}}{\mathcal{C}(\kappa / p)}\left\langle\psi_{C}^{-}(p)\left|T_{S C}\right| \psi_{C}^{+}(p)\right\rangle \\
& \simeq \frac{1}{1 / V_{S E}-J_{0}^{\overline{\mathrm{MS}}}}+\left(\frac{1}{1 / V_{S E}-J_{0}^{\overline{\mathrm{MS}}}}\right)^{2} \frac{V_{0}}{V_{S E}}\left[C_{001} \frac{\pi M}{12} \mu \kappa^{2}\left(\frac{V_{0}}{V_{S E}}-1\right)\right. \\
& -2 \pi C_{001} M \kappa^{3} \zeta^{\prime}(-2) \\
& \left.+\frac{M}{4 \pi}\left(A \kappa^{3}+B \kappa p^{2}\right) \frac{V_{0}}{V_{S E}}\right] \text {. }
\end{aligned}
$$

In solving the RG we kept only terms to first order in deviations from the fixed point. For consistency we should make the same approximation here, replacing $V_{S E}$ by $V_{0}$ in the second term of this expression. The first term in the square bracket then vanishes. The remaining terms in cancel if we make the choices,

$$
A=8 \pi^{2} C_{001} \zeta^{\prime}(-2), \quad B=0
$$

for the constants of integration in our momentum-dependent perturbation. The on-shell scattering amplitude then reduces to the same form, Eq. (21), as we obtained for the purely energy-dependent potential. This shows that the momentum-dependent eigenfunction of the RG equation is indeed an "equation of motion" term, affecting only the off-shell behaviour of the $T$ matrix. 


\section{DISCUSSION}

We have applied the RG to $S$-wave scattering by a combination of Coulomb and shortrange potentials, looking in particular at strongly interacting systems with bound states close to threshold. Unlike previous work [5, 6, 7, 8], our approach treats both energy- and momentum-dependence of the short-range potential separately. To handle the divergences generated by the contact interactions we use dimensional regularization with the PDS and $\overline{\mathrm{MS}}$ schemes to subtract the linear and logarithmic divergences. The RG equation is obtained by requiring that the off-shell scattering matrix be independent of the subtraction scale.

The resulting $R G$ equation has a physically interesting nontrivial solution. This is not an exact fixed point because it possesses a marginally relevant perturbation and so it evolves logarithmically with the regulator scale. Perturbations around this can be expanded in powers of energy, momenta and $\alpha$ (or the corresponding low-energy scale $\kappa=\alpha M / 2$ ). The RG eigenvalues and hence the power counting for purely energy-dependent perturbations are the same as those found for systems with only short-range interactions [10]. This reflects the fact that the Coulomb and free wave functions have the same power-law behavior near the origin. The fact that the Coulomb potential leads to a logarithmic discontinuity in derivatives of the wave functions is reflected by the presence of the marginal term proportional to $\kappa$.

Like the fixed point of the pure short-range potential, the nontrivial solution describes a system with a bound state at zero energy. The double expansion around it in powers of the energy and $\kappa$ corresponds to the Coulomb effective-range expansion originally introduced by Bethe [4], with the coefficients expanded in powers of $\alpha$.

We have extended this approach to terms that depend on the off-shell momenta, presenting in detail the analysis for the simplest of these, which is second-order in the momenta. The results for the power counting match with those found in the pure short-range case [10]. (Again, this is not surprising given the behavior of the Coulomb wave functions at the origin.) In particular, the momentum-dependent terms appear at higher orders than the corresponding energy-dependent ones. The one studied here has an RG eigenvalue $\nu=2$, compared with $\nu=1$ for the term proportional to the on-shell energy.

The structure of these momentum-dependent terms indicates that they arise from the equation of motion and hence they should affect only the off-shell behavior of the scattering 
matrix. We have verified that our second-order term does not contribute to scattering observables.

This RG analysis can explain some of the puzzling features seen in other applications of EFTs to charged-particle scattering. The different power counting for energy- and momentum-dependent perturbations around the nontrivial fixed point means that one cannot "use the equations of motion" to remove energy-dependence from the effective potential without mixing terms of different orders. Requiring the potential to be energy-independent for all values of the regulator scale is only possible if the higher-order momentum dependent perturbations are given unnaturally large coefficients. This is quite different from expansions around a trivial fixed point where the corresponding terms appear at the same order.

Most approaches to the EFT for charged-particle scattering are based on purely momentum-dependent potentials (derivatives of $\delta$-functions) [5, 6, 7]. Each term in such a potential can be built out of a combination of energy-dependent perturbations and off-shell ones. The leading-order piece of each is the energy-dependent one and so these potentials can reproduce the Coulomb effective-range expansion. However, as mixtures of different RG eigenfunctions, these terms contain pieces that violate the power counting. As a result, the inclusion of higher-order terms can significantly change the coefficients of lower-order ones.

Moreover, the same on-shell perturbation can form part of more than one distinct momentum-dependent term. For example, Ref. [7] shows that both the structures $k^{4}+k^{\prime 4}$ and $k^{2} k^{2}$ can generate a shape parameter in the effective-range expansion. However they contain different admixtures of off-shell perturbations and hence they affect the renormalization of the lower-order terms differently, as demonstrated by the numerical results in that paper.

Finally, the combination of momentum-dependent perturbations and the Coulomb potential leads to new divergences. These arise from derivatives of the Coulomb wave functions at the origin. We have used DR and PDS to regularize these but we found it necessary to keep their subtraction scale distinct from the one used for the integral over intermediate states. We regard this second scale as analogous to a factorization scale, separating off the nonperturbative regime caused by the singularity in the long-range interaction at the origin. Although we have been unable to obtain a useful RG equation by demanding that the off-shell scattering matrix be independent of this scale, we are able to show that physical observables do not depend on it. 


\section{Acknowledgments}

This work was supported by STFC grant number PP/F000448/1.

[1] S. R. Beane et al., in At the Frontier of Particle Physics: Handbook of QCD, edited by M. Shifman (World Scientific, Singapore, 2001), Vol. 1, p. 133.

[2] P. F. Bedaque and U. van Kolck, Ann. Rev. Nucl. Part. Sci. 52, 339 (2002).

[3] E. Epelbaum, Prog. Part. Nucl. Phys. 57, 654 (2006)

[4] H. A. Bethe, Phys. Rev. 76, 38 (1949).

[5] X. Kong and F. Ravndal, Phys. Lett. B450, 320 (1999).

[6] X. Kong and F. Ravndal, Phys. Rev. C 64, 044002 (2001).

[7] S. Ando, J. W. Shin, C. H. Hyun and S.-W. Hong, Phys. Rev. C 76, 064001 (2007).

[8] T. Barford and M. C. Birse, Phys. Rev. C 67, 064006 (2003).

[9] K. G. Wilson and J. G. Kogut, Phys. Rep. 12, 75 (1974).

[10] M. C. Birse, J. A. McGovern and K. R. Richardson, Phys. Lett. B464, 169 (1999).

[11] D. B. Kaplan, M. J. Savage, M. B. Wise, Phys. Lett. B 424, 390 (1998).

[12] D. B. Kaplan, M. J. Savage, M. B. Wise, Nucl. Phys. B 534, 329 (1998).

[13] R. Higa, H.-W. Hammer and U. van Kolck, arXiv:0802.3426. 